\title{
Fracture mode transitions during indentation of columnar TiN coatings on metal
}

\author{
S. BHOWMICK $\uparrow$, R. BHIDE + , M. HOFFMAN§, \\ V. JAYARAM ${ }^{* \dagger}$ and S. K. BISWAS \\ $\dagger$ Department of Metallurgy, Indian Institute of Science, Bangalore 560 012, India \\ $\$$ Multi-Arc India Ltd, Pune 411018, India \\ $\S$ School of Materials Science and Engineering, \\ The University of New South Wales, Sydney, New South Wales 2052, Australia \\ -Department of Mechanical Engineering, \\ Indian Institute of Science, Bangalore 560012, India
}

(Received 20 September 2004; in final form 18 February 2005)

\begin{abstract}
Pyramidal indentations and focused-ion-beam machining have been used to study the damage during contact loading of textured columnar titanium nitride hard coatings on three steels and aluminium. The influence of the substrate hardness and coating thickness on the type and extent of fracture have been examined. In particular, the primary mechanism of deformation is shown to undergo a change from inter-columnar shear to other forms of subsurface damage. A physical model is proposed to explain this transition in the fracture mode. The implications of such behaviour for coating design are discussed.
\end{abstract}

\section{Introduction}

Hard ceramic materials, such as titanium nitride (TiN), are usually applied as thin protective coatings on soft substrates to enhance the performance and mechanical reliability of components that include cutting tools, gears, high speed drills, dies and punches. Since these coatings are usually brittle, the life of the components is limited by the failure of films, which is mainly caused by stresses that arise because of contact loading in service. This kind of failure of the coating often involves initiation of cracks on the coating surface and propagation of these cracks through the coating material. Thus, knowledge of damage modes of the coating under contact loading is of major importance for the overall performance of a coated material in practical applications. We have, in this paper, attempted to arrive at a broad physical understanding of what drives damage in films with an industrially popular columnar and textured microstructure.

The mode of deformation under an indenter of a typical hard thin film such as TiN on a ductile substrate has generally been assumed to be dominated by the plastic deformation of the underlying substrate. Only for very shallow contact depths,

*Corresponding author. Email: qjayaram@met.iisc.ernet.in 
typically about $5-10 \%$ of the film thickness and in the case of a sharp tip indenter, is plastic flow of the coating expected to be the dominant mode of deformation [1]. In all other instances, the response of the film appears to be dominated by cracking. The commonly observed modes of fracture are the so-called nested cracks which lie parallel to the indenter edges at the surface [2-7] and the edge cracks which have recently been shown [8] to have very shallow trajectories and do not, in general, come close to the interface. The other two modes that are extensively reported are the so-called bending cracks, both those that originate from the interface and move upwards until they meet the surface compressive zone, as well as the cracks that appear at the corner of the indentation and propagate from the surface downwards. More recently, a more severe-looking form of failure [8] was reported, the so-called inclined crack which lies entirely subsurface below the impression and propagates at an angle to the indentation axis. In contrast with these modes of failure that are characterized by large crack openings, a completely different form of deformation has been noted, typically at lower indentation loads, namely the shear cracking (sliding) of TiN columns [4, 9-12]. This particular mode shows little or no crack opening and appears to be characterized by a phenomenon akin to grain-boundary sliding and enables the film and the substrate to deform compatibly, such that the applied load is partitioned between a central slipped region in which the load is supported by the substrate and an outer periphery of columns that continue to support the balance of the applied load [12].

More recently, in an attempt to analyse the evolution of damage in the coating, Richter [13] carried out Vickers indentations on TiN films on different substrates. The surface views of the indentations indicate that the nature and intensity of features strongly depend on the film thickness and substrate hardness. In our recent studies, it has been demonstrated from sections of indentations that TiN films of $1-2 \mu \mathrm{m}$ on stainless steel display shear cracking of columns [12], while thicker $5 \mu \mathrm{m}$ films display inclined and bending cracks [8]. In an example of another material system, the change in crack orientations and density with increase in film thickness has also been reported by Lee et al. $[14,15]$ for thick brittle films of silicon nitride $\left(\mathrm{Si}_{3} \mathrm{~N}_{4}\right)$ on a brittle quasiplastic substrate of $\mathrm{Si}_{3} \mathrm{~N}_{4}-30 \mathrm{wt} \% \mathrm{BN}$. The effect of substrate hardness on the damage behaviour in a coating of $\mathrm{Si}_{3} \mathrm{~N}_{4}$ of approximate thickness $250 \mu \mathrm{m}$ has also been investigated by Lee et al. [15]. A comparison of coatings on different substrates indicates that the tensile and shear stresses in the film increase strongly with increasing elastic mismatch between substrate and coating. This leads to an increase in the intensity of damage in the case of a film on a relatively soft substrate. Thus, the above papers suggest that film thickness and substrate hardness strongly influence the nature (and not just the magnitude) of the response of TiN films to contact loading.

It is interesting to note that clarity in characterizing the damage in coatings has come about largely when features were examined not only from above but also through cross-sections of indentations. The mechanism of column slippage, the shallow profiles of nested cracks and the very existence of inclined cracks have all been determined through sectional microscopy. The present paper seeks to address the role of substrate plasticity and film thickness to provide an improved understanding of contact-induced damage of columnar TiN films. Such understanding is 
important in optimizing the thickness of a coating, particularly when the choice of substrate is dictated by other considerations.

\section{Experimental procedure}

Cathodic arc evaporation was used to deposit TiN films on four different substrates, namely aluminium (Al), mild steel (MS), stainless steel (SS) and high-speed steel (HSS). The substrates were cleaned thoroughly with solvent and dried before being placed on a continuously rotating planetary holder inside the vacuum chamber which was evacuated to a base pressure of $1.3 \times 10^{-3} \mathrm{~Pa}\left(10^{-5} \mathrm{Torr}\right)$. Prior to deposition the substrates were heated to the deposition temperature of $350^{\circ} \mathrm{C}$ and then sputter cleaned with $\mathrm{Ar}^{+}$. A thin layer of titanium (about $50 \mathrm{~nm}$ ) was deposited on the substrates to improve the adhesion of the TiN film. Deposition of TiN was carried out in high-purity nitrogen at a pressure of $2.6 \mathrm{~Pa}$ (20 mTorr). A negative bias voltage of $150 \mathrm{~V}$ was applied to the substrates during deposition. The as-deposited structure of the film contains columns of about $0.2-0.5 \mu \mathrm{m}$ diameter. X-ray diffraction reveals a complete $\langle 111\rangle$ texture along the growth direction for all films studied in the present work.

Instrumented indentations were carried out with an ultramicroindentation system (Commonwealth Scientific and Industrial Research Oranization, Sydney, Australia) and a Vickers microindenter for a range of loads. Cross-sections of the film and substrate were prepared and imaged using a focused-ion-beam (FIB) mill (FEI P200) for characterization of the microstructure and deformation following indentation. A crater is milled in the selected region using a rough mill with a beam current of $6600 \mathrm{pA}$. Final milling on the wall of interest is carried out with a beam current of $1000 \mathrm{pA}$, followed by cleaning of the wall at $350 \mathrm{pA}$. Images of the contact zones of deformed films were captured at $70 \mathrm{pA}$ after tilting the sample by $45^{\circ}$. The hardness of the substrates A1, MS, SS and HSS are measured by Vickers microindentation to be $0.4 \mathrm{GPa}, 2.3 \mathrm{GPa}, 6.1 \mathrm{GPa}$ and $8.8 \mathrm{GPa}$ respectively. This equates to a variation in the substrate yield stress of the order of $0.13-2.9 \mathrm{GPa}$.

\section{Results}

The observations of contact-induced deformation of TiN films are presented in this section as a function of film thickness and substrate hardness. A detailed microstructural analysis has been carried out to understand the mechanism operating inside the TiN films under indentation loading. It is well established that nested cracks parallel to the edges of an indentation appear on the coating surface under a sharp tip Vickers indenter and concentric rings of cracks form under a spherical indenter irrespective of film thickness. A recent publication on contact-induced damage in thick TiN films [8] affirms that the features observed inside an impression on the coating surface may have a shallow depth profile and, thus, may not play any major role in the bulk damage of the film. Thus, our emphasis here lies not on surface views but on cross-sections of the microstructure. To facilitate the microstructural description of a large number of impressions, a schematic diagram of the morphology and orientation of all the damage modes is shown in figure 1, while the 


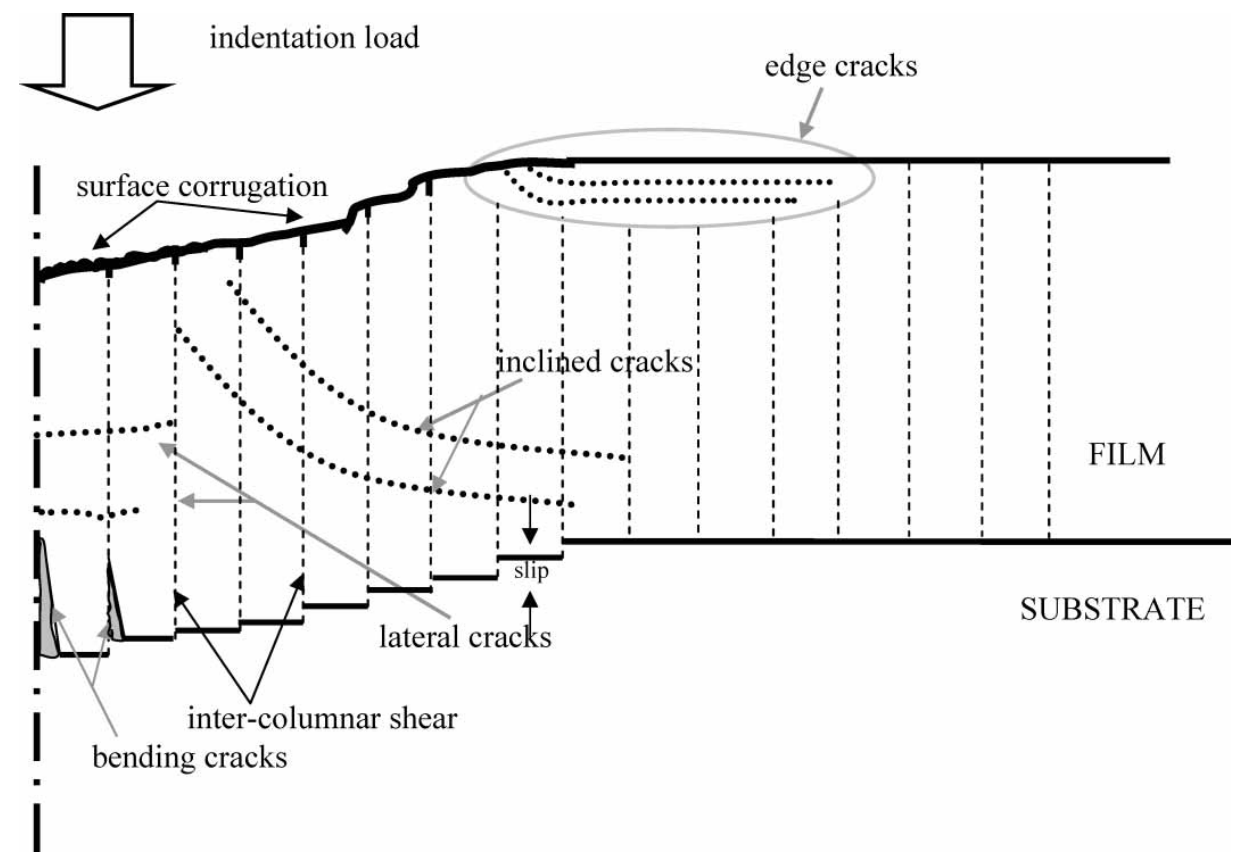

Figure 1. A schematic diagram showing regions of different modes of damage beneath a Vickers indentation (from [8]).

Table 1. Damage modes observed beneath indentations in TiN films as a function of substrate thickness and hardness. Major modes of damage are shown in bold.

\begin{tabular}{lllll}
\hline$t(\mu \mathrm{m})$ & \multicolumn{1}{c}{$\mathrm{Al}$} & \multicolumn{1}{c}{ MS } & \multicolumn{1}{c}{ SS } & \multicolumn{1}{c}{ HSS } \\
\hline 1.6 & Inclined cracks & Shear cracks & Shear cracks & Shear cracks \\
Bending cracks & Shear cracks & Shear cracks & Shear cracks \\
Bending cracks & & $\begin{array}{l}\text { Bending cracks } \\
\text { Inclined cracks }\end{array}$ & $\begin{array}{l}\text { Inclined cracks } \\
\text { Shear cracks, } \\
\text { Shear cracks }\end{array}$ & Shear cracks \\
& Inclined cracks & Lateral cracks & Inclined cracks & Shear cracks \\
& & & Bending cracks & \\
\hline
\end{tabular}

variation in principal damage modes are presented together in a tabular form in table 1 . On the basis of the features observed inside the films in the present system (four film thicknesses and four substrates of different hardness), the modes of damage may be broadly classified into two main categories. They consist of shear cracks along inter-columnar boundaries and trans-columnar cracks, which include inclined, lateral, edge and bending (as defined in figure 1). In addition, transcolumnar microcracks of the same length scale as those of individual columns are also seen normal to the indentation axis. The damage observed in the films of 
smallest thickness in the present systems will be presented first and then, considering the three substrates individually, the evolution of damage with increase in film thickness will be discussed.

A plan view of a typical impression on TiN films of approximate thickness $1.6 \mu \mathrm{m}$ on SS substrates is shown in figure $2 \mathrm{a}$. The impression reveals the appearance of continuous and discontinuous steps which have spacings similar to the intercolumnar spacing $(0.2 \mu \mathrm{m})$. Although the plan view of the impression does not reveal the nature of the cracks, a side view of the indentation (figure $2 b$ ) indicates that there is little crack opening, but a major component of shear, as evidenced by the relative vertical displacement of columns into the substrate which give rise to steps at the interface. The summation of step lengths at the deformed interface appears to be equivalent to the indenter displacement. Thus, the main mode damage in a TiN film on SS appears to be shear slippage along inter-columnar boundaries. Similar features are seen in $1.6 \mu \mathrm{m}$ films deposited on softer (MS) and harder (HSS) substrates.

In the case of HSS substrates, films across the entire thickness range reveal steps at the film-substrate interface from the indentation axis to the edge of an impression as shown in figure 3 for films of thickness 1.6, 3.5 and $5.9 \mu \mathrm{m}$. Thus, the main damage mechanism in TiN films on HSS can be considered as shear cracking along intercolumnar boundaries irrespective of film thickness. On the other hand, the crosssectional micrographs of the indented zone of films deposited on MS and SS reveal, with increasing film thickness, the appearance of another mode of damage inside the film, namely inclined cracks. As shown in figure 4 for MS, these cracks are usually inclined to the indentation axis and do not extend up to the free surface of the coating. They propagete mainly towards the film-substrate interface and tend to curve away from the substrate near interface. Other types of damage which are observed in cross-sectional views (figure 4) are bending cracks that initiate from the film-substrate interface and move towards the free (indented) surface, lateral cracks that originate within the film and run parallel to the interface. All these fracture modes, inclined, lateral and bending, appear to increase at the expense of the inter-columnar shear steps. The inclined cracks can be visualized as conical in profile in three dimensions. Although no direct evidence is obtained in the present study to specify the mode of propagation of inclined cracks, the contrast obtained in the FIB image reveals that there is a displacement of the columns across the crack as indicated in figure $4 \mathrm{c}$, which strongly suggests that, like the inter-columnar cracks, they propagate in mode II under shear. Note that columnar sliding steps at the interface are greatly reduced or absent in these thick films.

Thus, a significant finding in the present investigation, is the transition from one mode of fracture to another on increase in film thickness. The change from intercolumnar shear cracks to trans-granular inclined cracks occurs for MS and SS, between film thicknesses of about $1.6-3.5 \mu \mathrm{m}$ and $3.5-4.9 \mu \mathrm{m}$ respectively. For HSS substrates, as pointed out earlier, only shear steps are seen, suggesting that the critical thickness lies beyond the range studied here.

Next, the load dependence on the evolution of damage is described. Figure 5 illustrates the orientation of the cracks observed at different loads in a thick film $(5.9 \mu \mathrm{m})$ on SS. The features suggest that, at a very low load, small segments of columns, of the same width as the column diameter and almost parallel to the surface of the coating, develop cracks just below the face of the indenter (AB). 


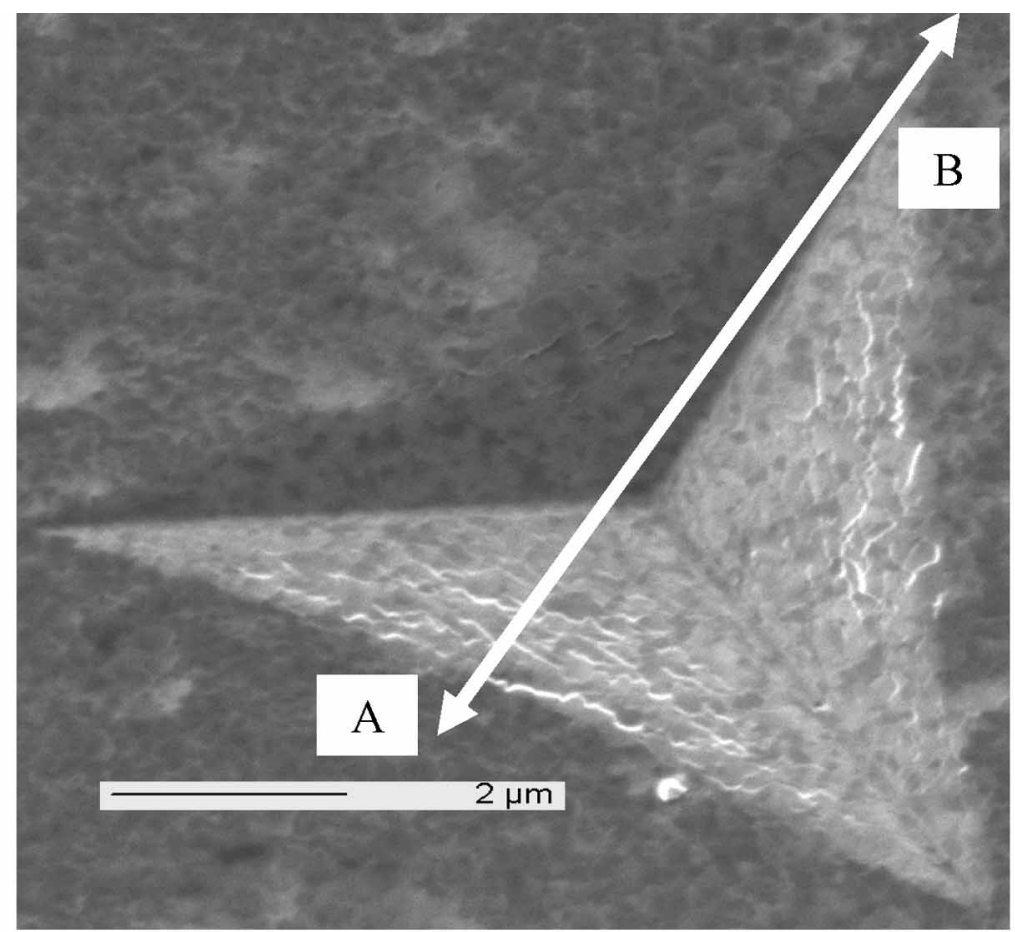

(a)

A

B

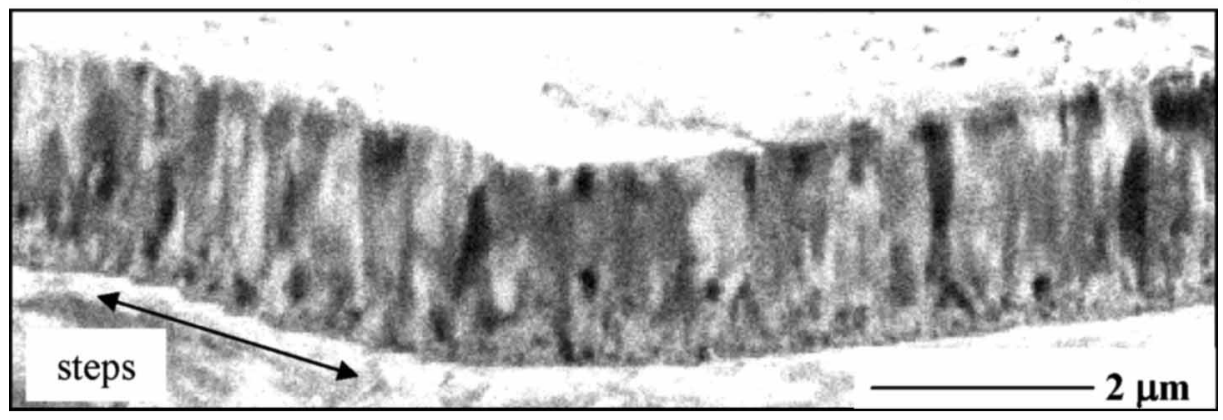

(b)

Figure 2. FIB images of a Berkovich impression on TiN film on SS at about $0.5 \mathrm{~N}$ load showing (a) a surface view with cracks parallel to the edges and (b) a cross-section showing steps at the film-substrate interface. The cross-section sample is prepared by milling the impression perpendicular to $\mathrm{AB}$.

A comparative examination of the cross-sectional images at high loads (figures 4 and 5) indicate that, as the load increases, the small cracks either propagate obliquely outwards from the indentation axis or link with each other and develop continuous inclined cracks inside the film. A few microcracks and inter-columnar shear cracks 


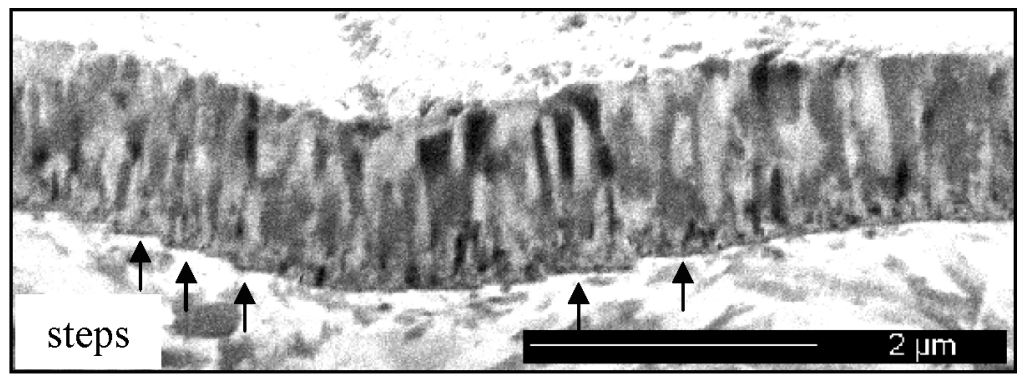

(a)

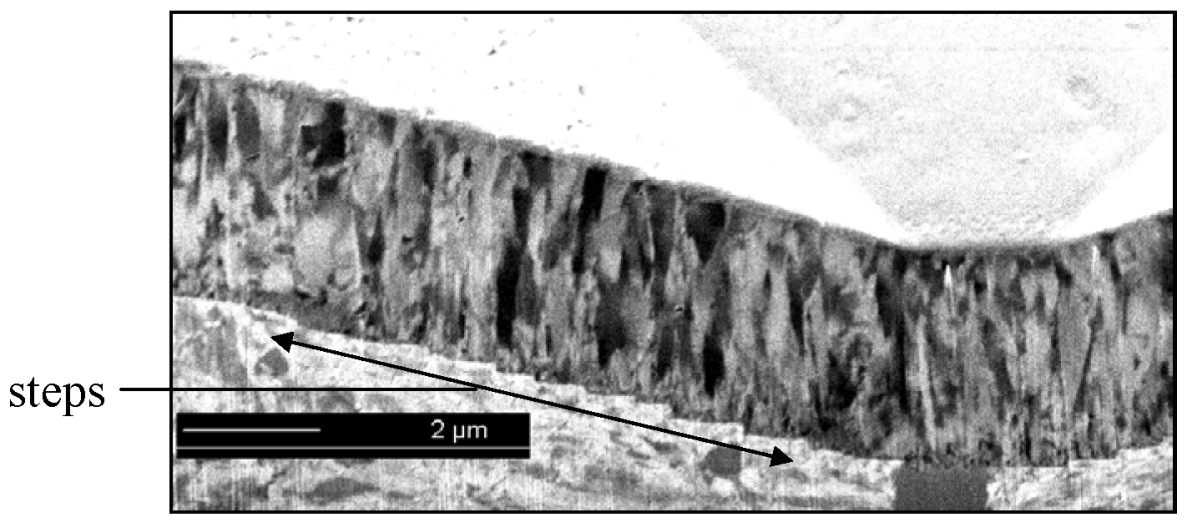

(b)

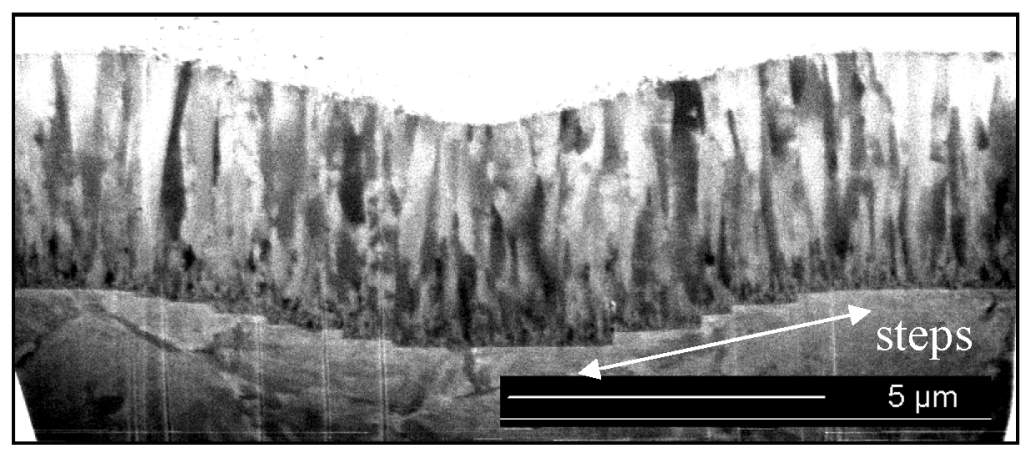

(c)

Figure 3. Cross-sectional FIB images of indentations of TiN films of thicknesses (a) $1.6 \mu \mathrm{m}$ (0.5 N load), (b) $4.9 \mu \mathrm{m}$ ( $5 \mathrm{~N}$ load) and (b) $5.9 \mu \mathrm{m}$ ( $2 \mathrm{~N}$ load) on HSS, showing steps at the film-substrate interface.

also appear near the interface at low loads (figure 5). However, at higher loads it has been noticed that the number of such cracks does not increase greatly. No evidence of interfacial delamination of the film has been seen in the present study. On one occasion, the microcracks that formed at low loads appeared to extend in both 


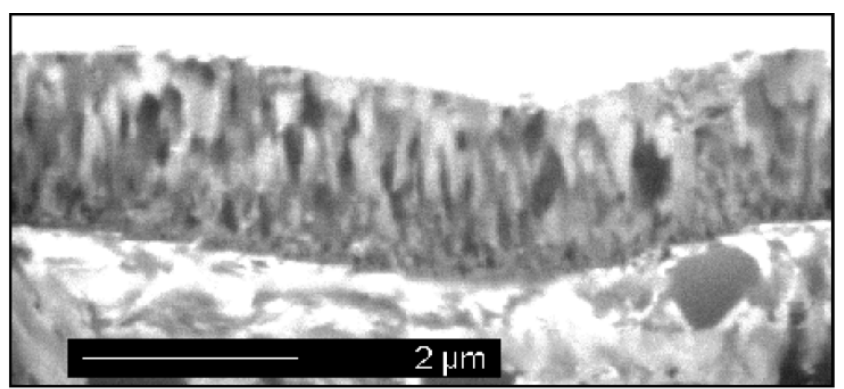

(a)

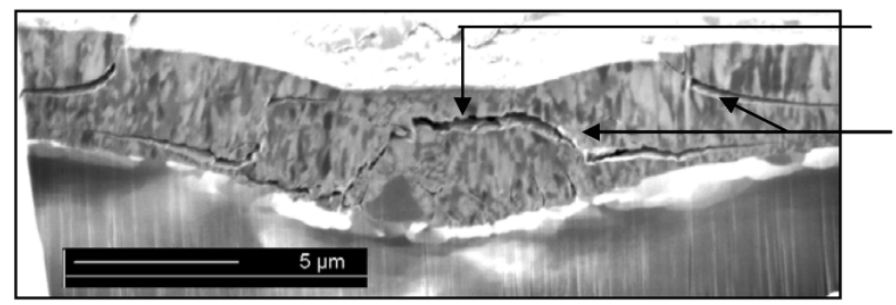

lateral crack inclined cracks

(b)

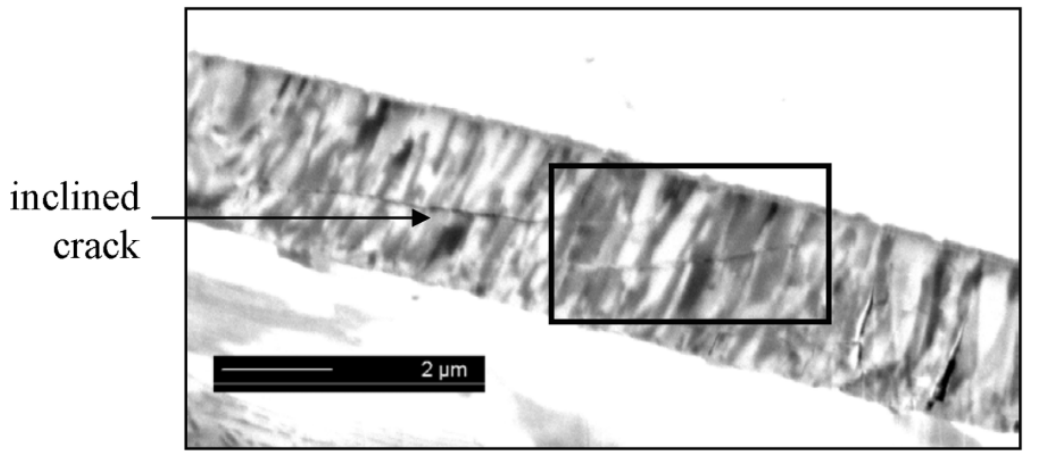

(c)

Figure 4. Cross-sectional FIB images of indentations of films of thicknesses (a) $1.6 \mu \mathrm{m}(0.3 \mathrm{~N}$ load), (b) $4.9 \mu \mathrm{m}$ ( $1 \mathrm{~N}$ load) and (c) $4.9 \mu \mathrm{m}$ ( $5 \mathrm{~N}$ load) on MS, showing inclined cracks. Shear steps in the thin film in (a) are replaced by inclined and lateral cracks in (b). The boxed region in (c) reveals lateral displacement of columns across the inclined cracks, consistent with shear.

directions at higher loads, joined the inclined crack and detached the upper part of the coating under indentation as indicated by the cross-sectional image (figure $4 \mathrm{~b}$ ).

In order to compare the damage features on the coating surface in the cases of three steel substrates, planar views of a high load indentation are displayed in figure 6. It may be noted that the extent of nested cracks in the centre with spacings of about a column diameter is a maximum for HSS and almost non-existent for MS, as supported by the cross-sectional observations. The zone of edge cracks at the periphery of the impressions with crack spacings of 5-10 $\mu \mathrm{m}$ expand in width from HSS to MS. However, as we have already shown, the major difference in the response of these films is only seen in the cross-sections, indicating the inadequacy 
A

B

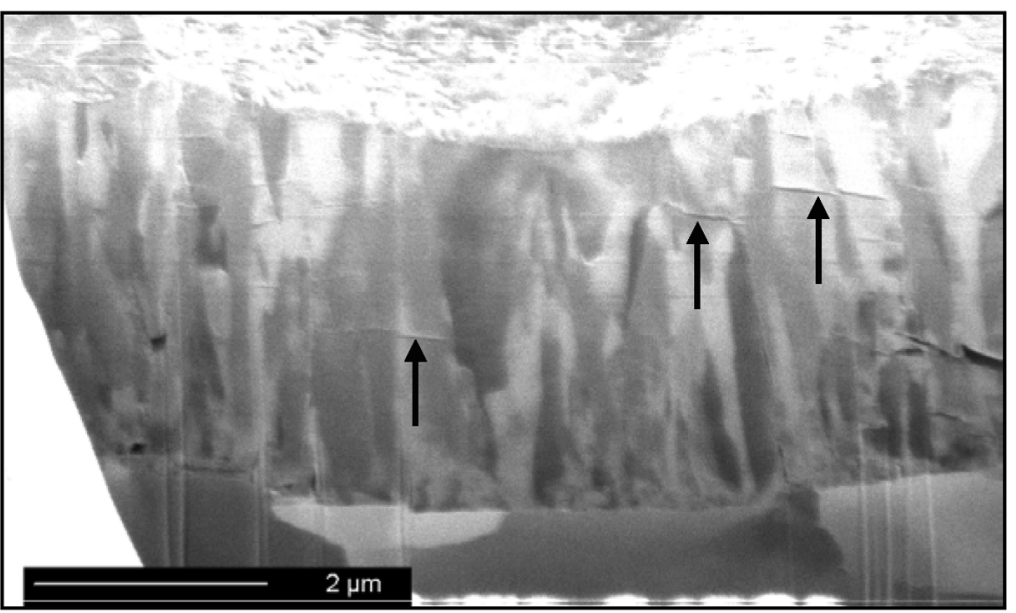

(a)

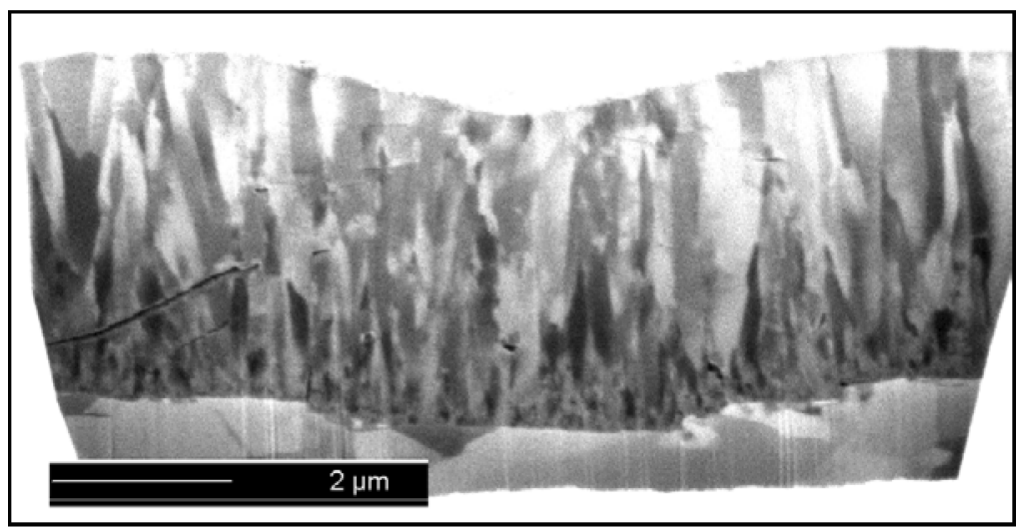

(b)

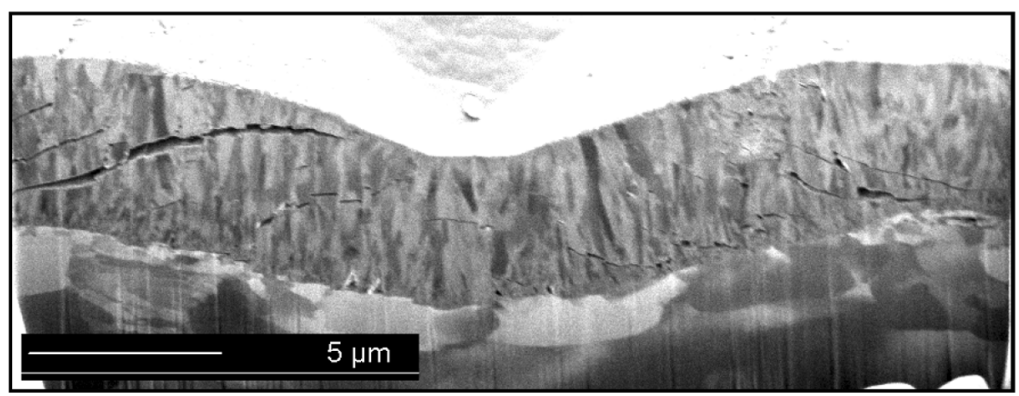

(c)

Figure 5. Cross-sectional FIB images of indentations on $5.9 \mu \mathrm{m}$ TiN on SS at loads of (a) $0.5 \mathrm{~N}$, (b) $1 \mathrm{~N}$ and (c) $5 \mathrm{~N}$, illustrating the development of transverse microcracks (arrowed in (a)) and inclined cracks in (b) and (c). 


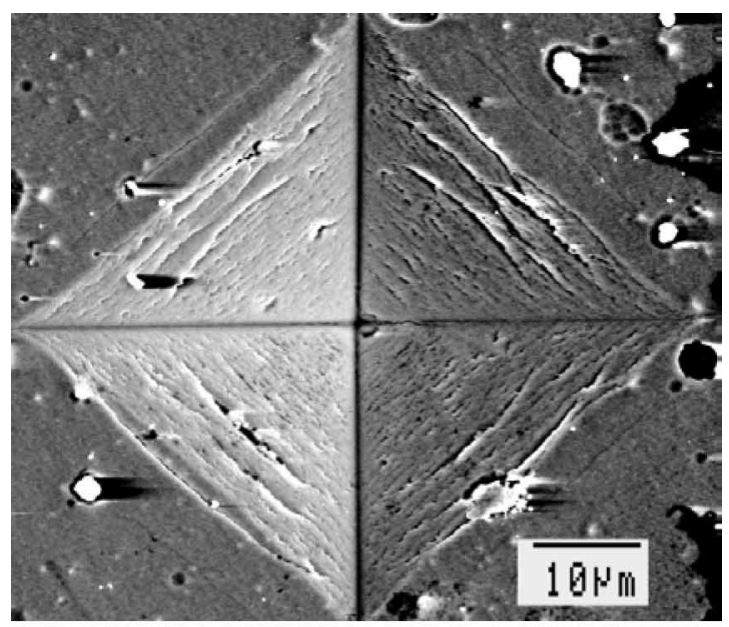

(a)

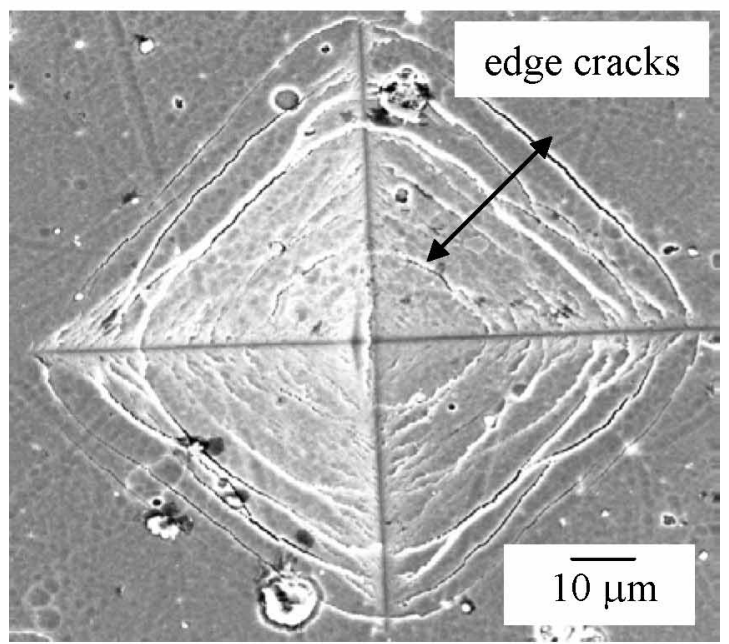

(b)

Figure 6. Features inside impressions (scanning electron microscopy) in films $4.9 \mu \mathrm{m}$ thick on (a) HSS and (b) MS at $10 \mathrm{~N}$ load. The regions of edge cracks are indicated (b).

of plan views to provide an accurate representation of the deformation of these coatings. Thus, plan views of the impression are inadequate to probe the extent of the most important modes of failure in these coatings.

Since the above evidence suggests that thicker films and softer substrates favour these grosser form of damage, it is also of interest in the present study to examine the evolution of damage in the systems where the substrate is very much softer than MS. An Al substrate is chosen for this purpose. Here, even the thinnest film of $1.6 \mu \mathrm{m}$ reveals an array of inclined cracks along with some bending cracks near the film-substrate interface (figure 7a). However, instead of curving away 


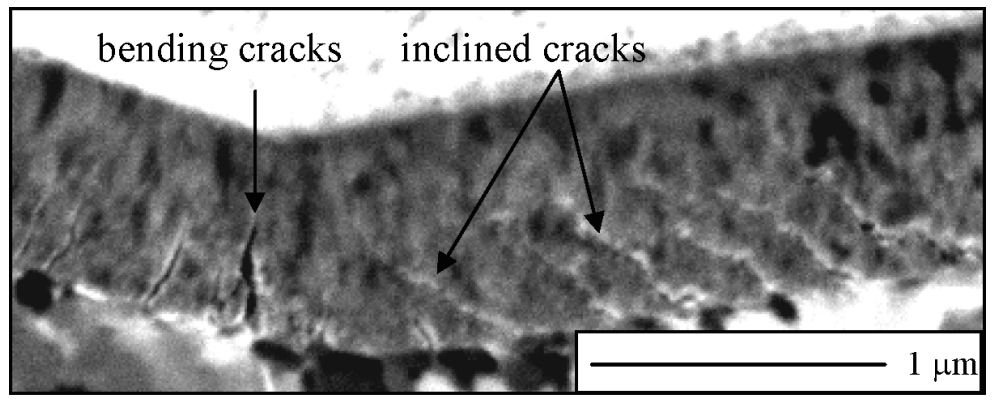

(a)

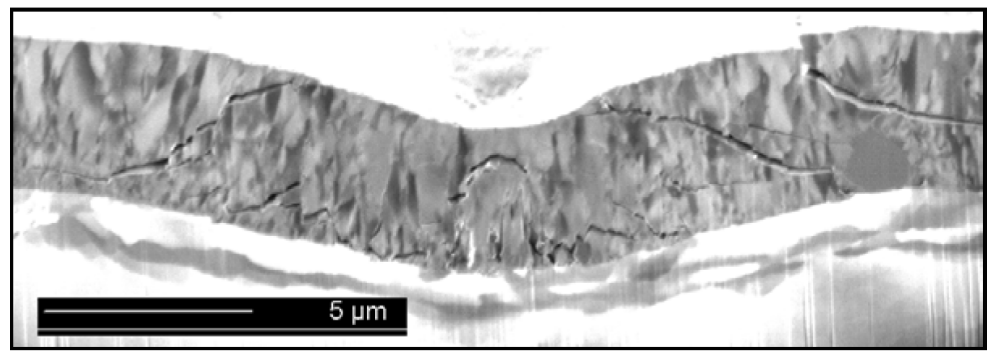

(b)

Figure 7. Cross-sectional FIB images of TiN films on Al: (a) $1.6 \mu \mathrm{m}$ film $(0.3 \mathrm{~N}$ load $)$ showing an array of inclined cracks near the interface; (b) $4.9 \mu \mathrm{m}$ film (1 N load) displaying inclined cracks and lateral cracks under indentation.

near the interface, as observed in the cases of MS and SS, the inclined cracks appear to run straight towards the substrate and to reach the interface. The inclined cracks together with other modes of damage, such as bending cracks and lateral cracks, which are also observed in thick films $(4.9 \mu \mathrm{m})$ deposited on $\mathrm{Al}$, are shown in figure $7 \mathrm{~b}$.

By comparing the trends in failure in systems with different substrate hardness and film thicknesses, a qualitatively unified picture of damage behaviour may be proposed, as shown in figure 8 . While substrates of intermediate hardness display a transition in fracture mode with increasing film thickness (between 1.6 and $3.5 \mu \mathrm{m}$ for MS and between 3.5 and $4.9 \mu \mathrm{m}$ for SS), the corresponding values for the hardest and softest substrates appear to lie outside the range used in the present investigation. Indeed, in the case of HSS it may be impossible to observe this transition practically, since coatings of very much greater thickness than $6 \mu \mathrm{m}$ are difficult to put down without spalling. Similarly, for a soft engineering material, such as Al, the transitions may lie in coatings in the nanometre range of thicknesses.

\section{Discussion}

The experimental observations indicate that the fracture response of columnar TiN films can be divided into two domains of behaviour. The first, typified by thin films 
film thickness

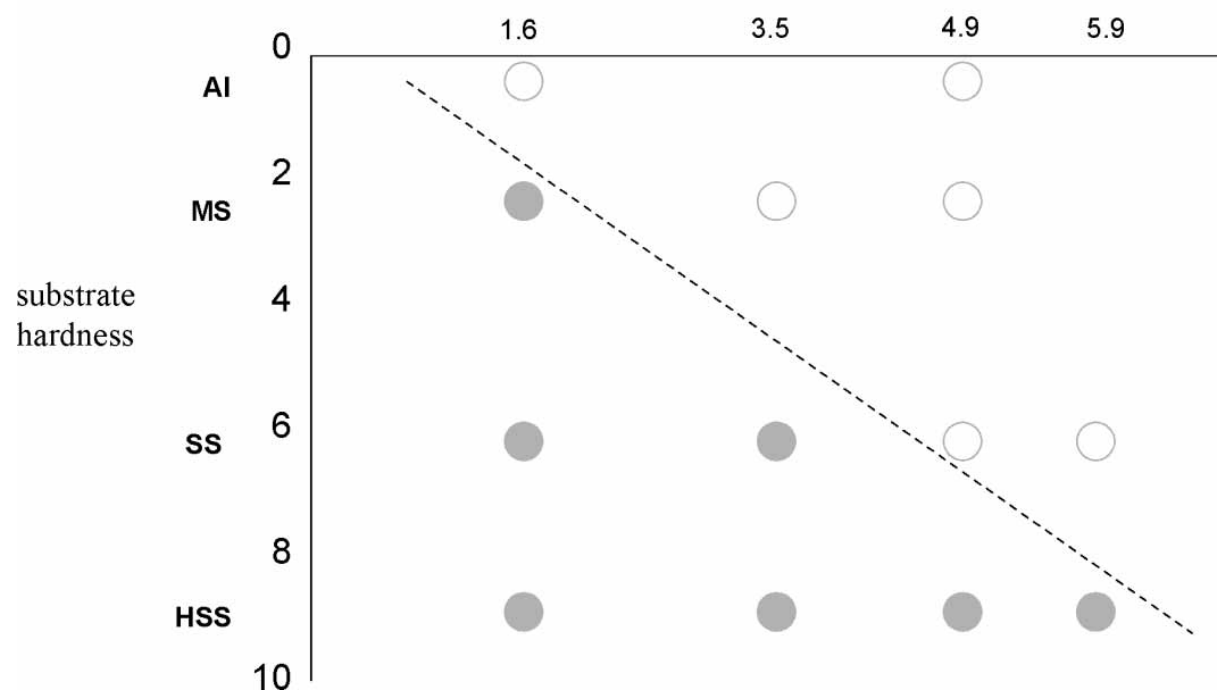

Figure 8. A schematic diagram showing the change in failure modes with film thickness and substrate hardness.

and hard substrates, is characterized by sensitivity to microstructure, while in the second class of systems (soft substrates and thick films) the cracks appear to be driven by the stress field and do not respect the columnar nature of the film. This combination of thickness and hardness is somewhat counter-intuitive. For a given load, the plastic deformation in a hard substrate will be small and the ensuing strains and damage in the film, which geometrically conforms to the deformed substrate, will be low. Keeping the film thickness constant, a softer substrate will deform to a greater extent and cause greater damage in the coating. Thus, to compensate, one might assume that a thicker film on the softer substrate would accommodate the strain energy to an equivalent extent. This is precisely what is experimentally not seen. In understanding this contrast, it is helpful to visualize the two options that a columnar film has, in deciding its response to contact loading, through the example of strong fibres, glued together and standing on a soft substrate. If the glue is weak, the fibres will simply slip past one another and sink into the substrate. The deformation in the fibres is now confined largely to the contact radius and the elastic-plastic zone in the substrate accommodates this amount of penetration. This model, which has been previously validated for TiN films on steel [1, 12] and which is briefly reproduced in figure 9, is characterized by a central slipped portion of the film through which a part of the load is supported by the elastic-plastic deformation of the substrate, and an outer periphery of columns that continue to support the remainder of the load since the low magnitude of normal pressure in that location has not yet caused fracture. Thus, the deformation of the film and substrate are compatible and indeed, by making this assumption of equality of displacements and 


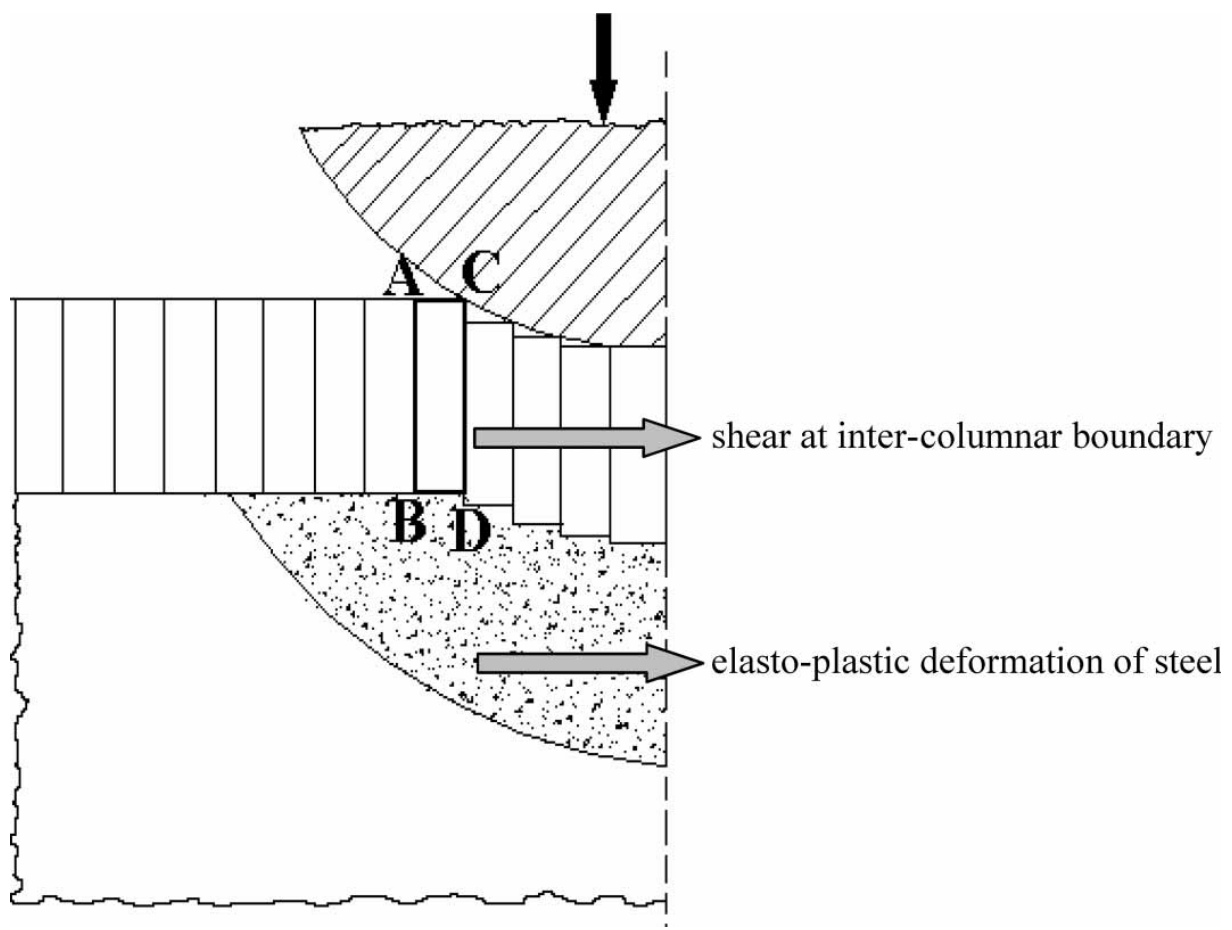

Figure 9. Schematic diagram of the deformation of the film-substrate system during intercolumnar shear. AB is the active inter-columnar boundary that supports a part of the total load. The remaining load is transferred to the substrate through the boundaries (including $\mathrm{CD})$ which have already undergone shear fracture.

modelling the system as consisting of two springs in parallel, the total load being the sum of the loads on each spring, it has been possible to reproduce accurately the load-displacement curve for columnar TiN films subjected to indentation when the mode of deformation is inter-columnar sliding. In this model, the inter-columnar shear strength emerges as an important material parameter in this model.

In contrast, when the column joints become strong and central slippage is not achieved, the system behaves as two springs in series where the same load acts on both, with the strains in each being dictated by their respective stiffnesses, and one may visualize the conventional indentation stress field to spread outwards from the contact until the stress at the substrate reaches the yield stress. Compatibility between the elastic columns and the plastic substrate is no longer assured since the interface is of a non-slipping kind, and one might expect a new phenomenon to emerge as a result of the stresses that must develop to take account of the strain mismatch.

We now apply these considerations to the problem at hand, that is the transition in deformation response with substrate hardness and film thickness. At a sufficiently low displacement, the film is known to respond according to the well-established expanding cavity model for indentation in an elastic-plastic material (figure 10). The interface, at this time, is remote from the deformation zone; the substrate does not 
(a)
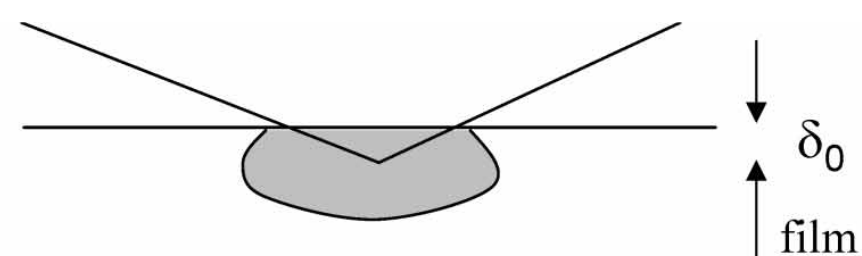

\section{substrate}

(b)

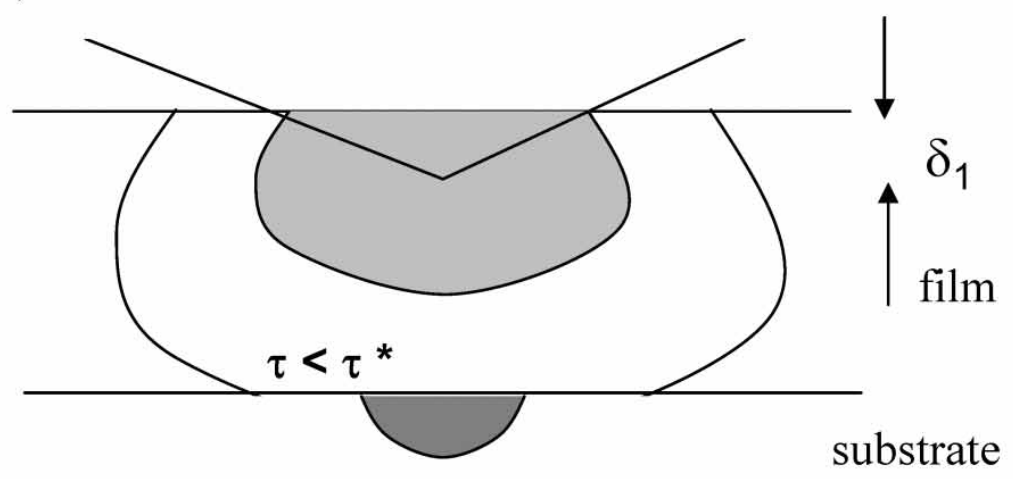

(c)

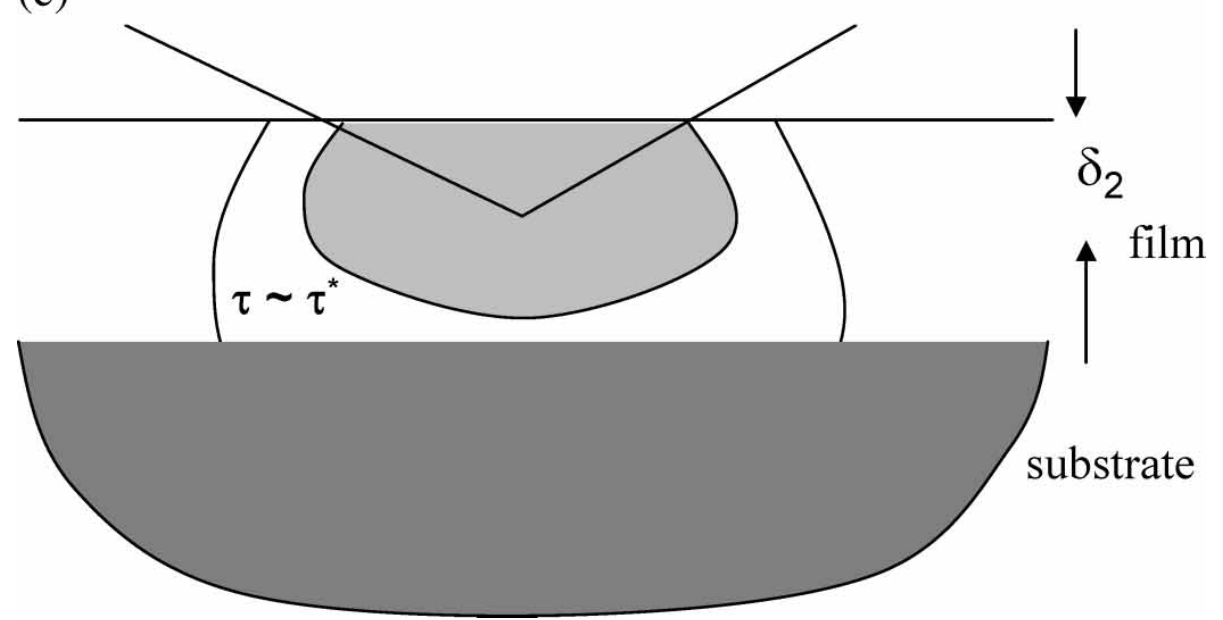

Figure 10. Schematic development of stresses and plastic zones as a fraction of indenter displacement $\delta$ : (a) low $\delta$, the film is effectively semi-infinite, and a plastic zone develops as in bulk TiN; (b) $\delta=\delta_{1}$, stresses initiate yielding in the substrate but $\tau$ (the shear stress on columns) $<\tau^{*}$ at the interface; (c) $\delta=\delta_{2}$, the plastic zone in the substrate expands further, $\tau \approx \tau^{*}$ and columnar sliding can take place. 
influence the film, nor is the stress in the film at the interface sufficient to cause sliding. As the displacement increases to a value $\delta_{1}$, the stress at the interface will rise until yielding can occur in the substrate (which, in the present work, is always softer than the film), as constrained by a film that continues to be elastic. As one continues to load, a second critical displacement $\delta_{2}$ will be attained, at which the stress at the interface in the film will also reach the critical value for columnar sliding. At this point the film can now exercise the option of slipping into the substrate by columnar sliding. This alternative is clearly preferable since this secondary indentation of the columns into the substrate acts as a flat punch with extremely high stresses at the concerns of the Boussinesq pressure field and concentrates the deformation in the contact area of the indentation, thereby allowing for a greater displacement of the indenter and, accordingly, enabling a greater amount of work to be performed by the applied load. In between these two critical displacements, the strains in the substrate become increasingly incompatible with those in the film. Thus, to summarize, the closer are the absolute magnitudes of $\delta_{1}$ and $\delta_{2}$, the greater is the likelihood that columnar shear will begin. Conversely, if $\delta_{1}$ and $\delta_{2}$ are widely separated, the strain mismatch is high and the resulting interfacial stresses can drive other modes of cracking, since columnar shear would not have commenced. It is easily seen that, if $\delta_{1}$ corresponds to a characteristic fraction of the film thickness (which will decrease as the substrate becomes softer), then, to minimize $\delta_{2}$ (which is desirable to promote inter-columnar shear at the expense of bulk fracture of the film), we need either a hard substrate or a thin film (and, of course, preferably both), in which eventuality the plastic zone in the substrate will not have expanded significantly by the time that the interfacial stress in the coating allows inter-columnar shear. In particular, the harder the substrate is in comparison with the intercolumnar shear strength, the greater is the allowable thickness. These ideas are substantiated by standard contact mechanical arguments from elastic deformation solutions; for example, as shown by Johnson [16], the axial strain generated during a displacement $\delta$ in a film of thickness $b$ deposited on a rigid substrate is proportional to $\delta / b$. Thus, a thin film allows a large stress to be developed at the film-substrate interface, thereby enabling column sliding to occur. We believe that the qualitative dependence of the stress with film thickness will not be greatly altered when the substrate is plastic.

In previous work $[1,12]$ we have shown that columnar sliding stresses in HSS, SS and MS are 3.5, 2.8 and 2.7 GPa respectively, leading to ratios of substrate hardness to columnar sliding stress of $2.5,2.0$ and 0.9 respectively. (These values are slightly different from the steels used in the present study but the present argument is not affected). This trend supports the present experimental observations that for a given thickness, such as 3.5 or $4.9 \mu \mathrm{m}$, the extent of columar shear decreases monotonically with hardness from HSS to Al. (Note that one might expect that columnar shear into a softer substrate would occur at a lower stress. However, the overriding consideration appears to be the larger interfacial stress that arises as a result of the low $\delta_{1}$.) Similarly, the hardest substrate (HSS) never displays the inclined, bending and lateral cracks, while the softest substrate always does, irrespective of film thickness in the approximate range $1.5-6 \mu \mathrm{m}$. A corollary of this analysis is that, if yielding in the substrate occurs at the same load as when the shear stress along the columns 
reaches the critical sliding stress, then the transition to non-sliding modes will never take place.

We have not undertaken to predict the exact mode of cracking that should follow from the above argument, since this would involve, most probably, a numerical analysis of the stress distribution around a contact, taking into account the existing anisotropy in microstructure. However, in this scenario, we suggest that the inclined cracks, lateral cracks and bending cracks are all consequences of this strain incompatibility between film and substrate and are driven by this mismatch stress field and not by the microstructure. Thus, the conditions that lead to columnar sliding also ensure compatible deformation and the least harmful mode of accommodation of the indentation load.

The implications for coatings design are many. In order to avoid the noncolumnar sliding modes of fracture, which are characterized by long cracks with large openings and which lend themselves to linkage and spalling, one would predict that each substrate would have a maximum permissible coating thickness. If this thickness needed to be increased, one would have to choose a harder substrate or to reduce the column sliding stress. One way of accomplishing the latter might be to exercise appropriate control of the deposition process to reduce the compressive residual stress in the film, which is suspected to influence the columnar sliding stress by a Coulombic type of friction law [1]. Thus, it is somewhat ironic that the thickest coatings can be supported by the substrates that might be least expected to need them, that is the hardest substrates. While the present analysis favours thin films, it is evident that there are loading conditions under which a thick film may be needed either because material loss is by an incremental process (such as erosion or corrosion) or because deformation of the contact surface would become too severe. In such situations a thick film cannot be avoided, but the maximum useful thickness may now be limited by the damage that results from the mechanisms described in the present work. Thus, for cutting tools in which $\mathrm{TiN}$ is deposited on a relatively hard substrate, one can afford to have thick coatings to provide a long life that is determined by a process of incremental wear. On the other hand, for a soft metal for which the coating primarily provides environmental protection and catastrophic damage under occasional impact is not permissible, the correct (and somewhat counter-intuitive) solution might well be to provide a thin film that will not crack.

While we have argued that the inclined and other grosser forms of cracks are not sensitive to the microstructure, there are indications that the columns provide microcrack nuclei that eventually propagate or link up to form the larger cracks. The observations of cracks of about $0.2-0.5 \mu \mathrm{m}$ (the column diameter) that are normal to the loading axis suggest the following mechanism, particularly in thick films in which the columns gradually thicken as the film grows and do not extend in a straight line from interface to surface. As illustrated in figure 11, the presence of a kink in a column requires that sliding is accompanied by a relative movement of opposite sides of the kink. Depending on the direction of the kink, this translation will lead either to microcracking or to two sides of a grain being forced into one another. In the latter situation, sliding may be prevented, while in the former case the sliding stress will be increased relative to the value seen for thin films with straight columns. This increase in sliding stress due to the energy needed to generate these microcracks may 


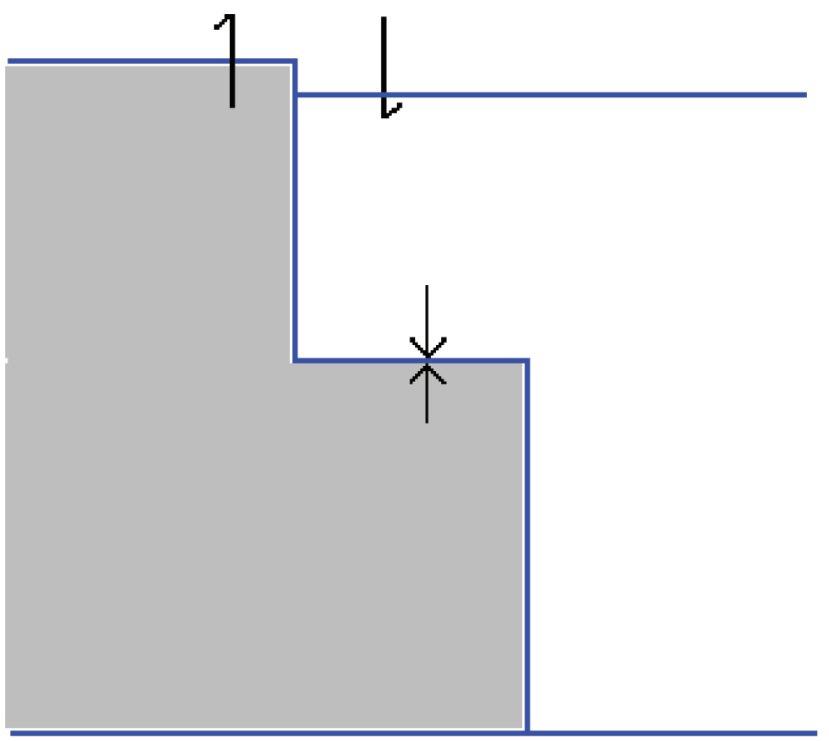

(a)

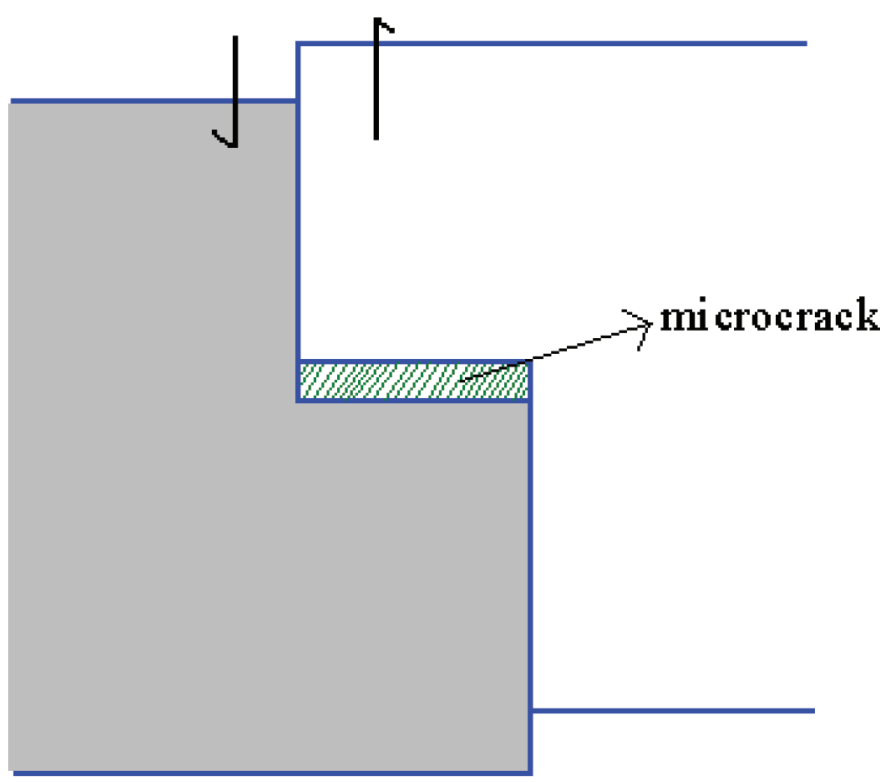

(b)

Figure 11. Schematic diagrams of the sliding mechanism to initiate transverse microcracks as seen in figure 5. A kink in the columns leads to a transverse section of a grain boundary which, depending on the sign of shear, can lead to (a) grain-grain interpenetration or (b) grainboundary cracking. 
also contribute, in thicker films, to the reduced tendency for inter-columnar shear cracks and the preference for other modes of damage.

\section{Conclusions}

Contact damage induced around indentations on TiN-coated metallic substrates indicates that the film undergoes a transition, with thickness and substrate hardness, from a relatively benign mode of inter-columnar sliding to more catastrophic modes of subsurface cracking. Hard substrates and thin films promote the former response, while thick films on soft substrates exhibit severe damage. It is shown that this transition can be understood from the standpoint of interfacial stresses that build up in the film as a consequence of two factors: the development of plasticity in the substrate and the inability of TiN columns to slide because the stress in the film at the interface has not exceeded a critical value for inter-columnar shear. For substrates of intermediate hardnesses, that is MS and SS, this transition occurs in the vicinity of about $2 \mu \mathrm{m}$ and $4 \mu \mathrm{m}$ respectively. The hardest steel always deformed by columnar shear while none of the films on soft Al did so, implying that the critical thicknesses for these two materials lay outside the range studied. Based on this type of behaviour, it is shown that for certain applications a thin columnar hard coating may be superior to a thick coating.

\section{Acknowledgements}

Financial support for this work was made possible by grants from the Defense Research and Development Organisation, Government of India, and the Australian Research Council Discovery Grant Programme. The assistance of Dr Z.-H Xie of the School of Materials Science and Engineering, University of New South Wales, in preparing the FIB samples is gratefully acknowledged.

\section{References}

[1] S. Bhowmick, V. Jayaram and S.K. Biswas, Acta Mater. 532459 (2005).

[2] J.C. Knight, T.F. Page and I.M. Hutchings, Surf. Engng 5213 (1989).

[3] E.R. Weppelmann, X.Z. Hu and M.V. Swain, J. Adhesion Sci. Technol. 8611 (1994).

[4] M. Shiwa, E.R. Wepplemann, A. Bendeli, M.V. Swain, D. Munz and T. Kishi, Surf. Coatings Technol. 68-69 598 (1994).

[5] M.V. Swain and J. Mencik, Thin Solid Films 253204 (1994).

[6] E. Weppelmann and M.V. Swain, Thin Solid Films 286111 (1996).

[7] J.S. Wang, Y. Sugimura, A.G. Evans and W.K. Tredway, Thin Solid Films 325163 (1998).

[8] S. Bhowmick, A.N. Kale, V. Jayaram and S.K. Biswas, Thin Solid Films 436250 (2003).

[9] E. Weppelmann, M. Wittling, M.V. Swain and D. Munz, Fracture Mechanics of Ceramics, Vol. 12, edited by R.C. Bradt (Plenum, New York, 1996).

[10] K.J. Ma, A. Bloyce and T. Bell, Surf. Coatings Technol. 76297 (1995).

[11] K.J. Ma, A. Bloyce, R.A. Andrievski and G.V. Kalinnikov, Surf. Coatings Technol. 94-95 322 (1997).

[12] S. Bhowmick, Z.-H. Xie, M. Hoffman, V. Jayaram and S.K. Biswas, J. Mater. Res. 192616 (2004). 
[13] J. Richter, Surf. Coatings Technol. 162119 (2003).

[14] K.S. Lee, S.K. Lee, D.K. Kim and B.R. Lawn, J. Am. Ceram. Soc. 812394 (1998).

[15] S.L. Lee, S. Wuttiphan, X. Hu, S.K. Lee and B.R. Lawn, J. Am. Ceram. Soc. 81571 (1998).

[16] K.L. Johnson, Contact Mechanics (Cambridge University Press, Cambridge, 1985). 